\title{
Revision Surgery for a Rod Fracture with Multirod Constructs Using a Posterior-Only Approach Following Surgery for Adult Spinal Deformity
}

\author{
Yu Yamato ${ }^{1,2}$, Tomohiko Hasegawa ${ }^{1}$, Go Yoshida ${ }^{1}$, Tomohiro Banno ${ }^{1}$, Shin Oe ${ }^{1,2}$, \\ Hideyuki Arima ${ }^{1}$, Yuki Mihara ${ }^{1}$, Hiroki Ushirozako ${ }^{1}$, Tomohiro Yamada ${ }^{1}$, Yuh Watanabe ${ }^{1}$, \\ Koichiro Ide ${ }^{1}$, Keiichi Nakai ${ }^{1}$, Kenta Kurosu ${ }^{1}$, Yukihiro Matsuyama ${ }^{1}$ \\ ${ }^{1}$ Department of Orthopaedic Surgery, Hamamatsu University School of Medicine, Hamamatsu, Japan \\ ${ }^{2}$ Division of Geriatric Musculoskeletal Health, Hamamatsu University School of Medicine, Hamamatsu, Japan
}

Study Design: Single-center retrospective case series.

Purpose: We aimed to evaluate the clinical results of revision surgery for a rod fracture using a posterior-only approach and determine the best procedure to prevent refracture in patients with adult spinal deformity (ASD).

Overview of Literature: ASD affects the thoracolumbar spine and often requires surgical correction. However, surgery for extensive spinal fusion causes rod fracture, a major mechanical complication. Few studies have described the treatment methods for rod fractures. Furthermore, the clinical outcomes of revision surgery for rod fractures in patients with ASD are currently unclear.

Methods: We retrospectively reviewed the medical records of 404 patients who underwent corrective fusion surgery for ASD with a minimum 2-year follow-up. We studied cases of reoperation for postoperative rod fractures and investigated surgical procedure, intraoperative findings, clinical course, and rod refracture following revision surgery.

Results: Rod fracture was observed in 88 patients (21.8\%). Fifty-three patients (average age, 68.3 years; average blood loss, 502.2 $\mathrm{mL}$ [\% estimated blood volume=16.4\%]; and operation time, 203.3 minutes) who suffered from a rod fracture at an average of 28.3 months after the primary operation underwent reoperation. Surgical invasiveness had no significant differences in total or partial rod replacement; however, the procedures with and without an anterior bone graft significantly differed. The replaced rod refractured at an average of 35.3 months after the revision surgery of five patients. The rod also refractured at a level outside multiple rods in two patients and with traumatic episodes in three patients. Three patients had bone grafts in the anterior column.

Conclusions: Revision surgery involving a multirod with a posterior-only approach for a rod fracture that occurred after ASD was performed successfully. Bone grafting in the anterior column is unnecessary for patients without massive bone defects.

Keywords: Rod fracture; Adult spinal deformity; Multirod constructs; Implant-related complications; Reoperation

\section{Introduction}

Adult spinal deformity (ASD) is a complex disorder that affects the thoracolumbar spine and often requires surgical correction, which can cause mechanical complications, particularly implant-related complications such as proxi-

\footnotetext{
Received Jun 12, 2021; Revised Aug 27, 2021; Accepted Sep 1, 2021

Corresponding author: Yu Yamato

Department of Orthopaedic Surgery, Hamamatsu University School of Medicine, 1-20-1, Handayama, Higashi-ku, Hamamatsu city, Shizuoka, 431-3192, Japan

Tel: +81-53-435-2299, Fax: +81-53-435-2296, E-mail: yamato@hama-med.ac.jp
} 
mal or distal junctional failure, screw pullout, and rod fracture [1-3]. The incidence of implant-related complications is $31.7 \%$ [1], which remains an unsolved problem. One of the major mechanical complications following surgery for extensive spinal fusion (especially regarding fusion to the pelvis) is rod fracture [4-9]. Many studies have reported a high incidence of rod fracture although few have described the treatment methods for rod fracture $[10,11]$. Indications for reoperation vary depending on the given study and surgeons $[10,11]$. Rod fractures are mainly caused by osseous nonunion [12]. Therefore, rods may fracture again after reoperation for rod replacement or reinforcement. However, studies have yet to clarify whether reoperation can alleviate the symptoms caused by fractured rods. Furthermore, the clinical course following rod fracture reoperation is unclear.

Reoperation can be performed using various surgical techniques such as replacement of all rods, partial replacement, reinforcement with connectors, bone graft in the anterior nonunion site, and multirod constructs. Luca et al. [10] reported a case series of patients who underwent revision surgery after the failure of pedicle subtraction osteotomy with rod fractures. They preferred cage insertion to reconstruction of the anterior column by adopting a combined posteroanterior approach or a lateral combined approach. We usually perform revision surgery via a single posterior approach with multirod constructs. This study aimed to evaluate the clinical results of reoperation using a single posterior approach for rod fracture and determine the best procedure that could prevent refracture.

\section{Materials and Methods}

This study was conducted in compliance with the principles of the Declaration of Helsinki. The study's protocol was reviewed and approved by the institutional review board of of Hamamatsu University School of Medicine (approval no., 20-107). The need for informed consent was waived because of the retrospective nature of the study. Data were retrospectively collected from a prospectively maintained database containing the operation records of 404 patients who underwent surgery for ASD at the University Hospital, Hamamatsu University School of Medicine between June 2010 and March 2018 and had a minimum 2-year follow-up. ASD was defined as the presence of at least one of the following indicators: degenerative or idiopathic scoliosis, congenital deformity, deformity with posttraumatic fracture, or iatrogenic deformity. Patients with ASD who underwent reoperation for rod fracture were included in this study. They were reoperated for rod fracture depending on their symptoms and request [11].

The following demographic variables were obtained: age, sex, perioperative surgical data of primary and revision surgeries, intraoperative bleeding, operating time, number of levels fused, fusion to the pelvis, and rod fracture site. The presence or absence of injuries that might have occurred when the rod was fractured was evaluated separately. Additionally, daily living activities, injuries such as falls, and absence of injuries were included. Surgical procedures and intraoperative findings on bone union were examined.

The clinical course of the symptoms and rod refracture after revision surgery was investigated through plain radiography. A rod fracture was defined as a fracture of the rod identified with radiography regardless of the presence of symptoms, including fractures incidentally detected on the routine examination of a radiograph. The patients underwent plain radiography when they visited the hospital for follow-up. The bone union of the anterior spinal column was assessed using three-dimensional computed tomography (CT) scans before and after rod fracture development. The upper and lower connection sites between the endplate or the surface of the vertebral osteotomy and grafted bone in coronal and sagittal views were used to evaluate the bone union at the three-column osteotomy and interbody fusion levels. Osseous union was defined as continuities in all four sites [11].

\section{Operative procedures of revision surgery}

Rod fractures were subjected to reoperation based on the following concepts: (1) using a posterior-only approach, (2) correction only by intraoperative body positioning, (3) bone transplantation to the interbody or vertebral body of the osteotomy site in patients with obvious bone defects on CT, (4) replacement of all the rods when connecting them in a series at the breakage site is difficult, (5) elongation of the fusion to the proximal side of patients with symptomatic proximal junctional kyphosis, and (6) application of multirod constructs in all patients. Revision surgery was performed by combining these procedures in duplicate. Bone grafting to the interbody or osteotomy site was performed as follows: the scar tissue was dissected from a posterior approach; the disc space and osteotomy 
site were expanded and curetted; and nonstructural bone grafting was performed in the gap of the bone because of insufficient space. An autogenous bone from the lamina or spinous process was used for the graft. Bone union was also evaluated during revision surgery. The mobility of the fused segment was examined while applying direct passive stress to the screw with the rod removed. Osseous nonunion was defined as obvious mobility at the rod fracture site.

\section{Statistical analysis}

Demographic data were subjected to descriptive statistics, including means and standard deviations. Paired $t$-tests were performed to examine the differences in spinopelvic parameters before and after revision surgery. Unpaired $t$-tests were conducted to compare the differences in surgical parameters between surgical procedures. Data were statistically analyzed using EZR ver. 2.6-1 (Saitama Medical Center, Jichi Medical University, Saitama, Japan), which is a graphical user interface for $\mathrm{R}$ ver. 3.6.1 (The $\mathrm{R}$ Foundation for Statistical Computing, Vienna, Austria). It is a modified version of the $\mathrm{R}$ commander designed to add statistical functions frequently used in biostatistics [13]. Results with $p<0.05$ were considered statistically significant.

\section{Results}

Rod fractures were observed in 88 of the 404 patients with ASD, and the incidence of rod fractures was $21.8 \%$. A total of 53 patients (12 men and 41 women; average age, 68.3; range, $41-84$ years) were reoperated for rod fractures. The average follow-up period, including the first surgery to the final follow-up, was 70.1 months (range, 24-120 months). Table 1 shows the demographic data, including primary operative parameters, of patients who underwent reoperation for rod fractures. After the primary operation, five patients underwent an extension to the ilium with a rod exchange because of distal junctional kyphosis. A total of 50 patients had rods fused to the ilium with iliac screws before rod fracture. Besides, three patients underwent proximal extension for a proximal junctional failure. The corresponding reoperation was performed at an average of 28.3 months (range, 5-62 months) after the primary operation through which the fractured rod was installed.

The average amount of blood loss and the duration of
Table 1. Demographic characteristics

\begin{tabular}{|c|c|}
\hline Characteristic & Value \\
\hline No. of patients & 53 \\
\hline Age (yr) & $68.3 \pm 9.2$ \\
\hline \multicolumn{2}{|l|}{ Sex } \\
\hline Male & 12 \\
\hline Female & 41 \\
\hline \multicolumn{2}{|l|}{ Initial operation } \\
\hline Operation time (min) & $428.8 \pm 84.7$ \\
\hline Blood loss (mL) & $1,860.0 \pm 1,141.3$ \\
\hline$\%$ Estimated blood volume (\%) & $60.0 \pm 38.0$ \\
\hline \multicolumn{2}{|l|}{ Upper instrumented vertebra } \\
\hline T3 & 1 \\
\hline T4 & 8 \\
\hline T5 & 3 \\
\hline T6 & 1 \\
\hline $\mathrm{T7}$ & 2 \\
\hline T8 & 6 \\
\hline T9 & 9 \\
\hline $\mathrm{T} 10$ & 23 \\
\hline \multicolumn{2}{|l|}{ Lower instrumented vertebra } \\
\hline $\mathrm{T} 12$ & 1 \\
\hline L1 & 1 \\
\hline L3 & 1 \\
\hline S1 & 3 \\
\hline Ilium & 47 \\
\hline No. of fusion levels & $9.8 \pm 2.4$ \\
\hline Duration between initial and revision surgery (mo) & $28.3 \pm 15.0(5-62)$ \\
\hline \multicolumn{2}{|l|}{ Radiofrequency site } \\
\hline PLIF/TLIF & 13 \\
\hline Posterolateral lumbar fusion & 15 \\
\hline Lateral lumbar interbody fusion & 1 \\
\hline Pedicle subtraction osteotomy & 15 \\
\hline Posterior vertebral column resection & 6 \\
\hline Sacroiliac joint & 3 \\
\hline \multicolumn{2}{|l|}{ Rod material } \\
\hline Cobalt chromium & 3 \\
\hline Titanium alloy & 26 \\
\hline Commercially pure titanium & 24 \\
\hline \multicolumn{2}{|l|}{ No. of rod } \\
\hline 2 & 40 \\
\hline 3 & 10 \\
\hline 4 & 3 \\
\hline
\end{tabular}


Table 1. Continued

\begin{tabular}{lc} 
Characteristic & Value \\
Present symptom & \\
\hline Back or low back pain & 46 \\
\hline Buttock pain & 7 \\
\hline Lower leg pain & 3 \\
\hline None & 5 \\
\hline Injury situation & \\
\hline None & 15 \\
\hline Life action & 34 \\
\hline Trauma & 3 \\
\hline Unknown & 1 \\
\hline Radiographic parameters & \\
\hline Preoperative & \\
\hline PI-LL $\left(^{\circ}\right)$ & $46.1 \pm 24.3$ \\
\hline PT ( $\left.{ }^{\circ}\right)$ & $37.8 \pm 10.8$ \\
\hline SVA (mm) & $142.5 \pm 80.8$ \\
\hline Postoperative & \\
\hline PI-LL $\left(^{\circ}\right)$ & $12.1 \pm 12.3$ \\
\hline PT $\left(^{\circ}\right)$ & $22.6 \pm 8.6$ \\
\hline SVA $(m m)$ & $47.1 \pm 49.3$ \\
\hline
\end{tabular}

Values are presented as number, mean $\pm S D$, or mean $\pm S D$ (range), unless otherwise stated.

PLIF/TLIF, posterior lumbar interbody fusion/transforaminal lumbar interbody fusion; PI, pelvic incidence; LL, lumbar lordosis; PT, pelvic tilt; SVA, sagittal vertical axis; SD, standard deviation. revision surgeries following rod fracture were $502.2 \mathrm{~mL}$ (\% estimated blood volume $=16.4 \%$ ) and 203.3 minutes, respectively (Table 2). The average follow-up period, including revision surgery until the final follow-up, was 46.7 months (range, 3-92 months). Two of the patients were lost to follow-up within 1 year. The symptoms following rod fracture development were alleviated immediately after the revision surgery of all patients. The number of patients who underwent each reoperative procedure is presented in Table 2. The levels of anterior bone grafting were

Table 2. Operative procedure in reoperation for rod fractures ( $N=53$ cases)

\begin{tabular}{|cc|}
\hline Variable & Value \\
\hline Operation time (min) & $203.3 \pm 63.7$ \\
\hline Blood loss (mL) & $502.2 \pm 477.5$ \\
\hline$\%$ Estimated blood volume (\%) & $16.4 \pm 16.6$ \\
\hline Re-operative method & 42 \\
\hline Total rod replacement & 11 \\
\hline Partial rod replacement & 21 \\
\hline Bone graft in interbody or osteotomy site & 12 \\
\hline Elongation to proximal (for PJK) & 53 \\
\hline Multi-rod construct & 46.7 (3-92) \\
\hline Follow-up period after revision (mo) & 5 \\
\hline Re-fracture & \\
\hline
\end{tabular}

Values are presented as mean \pm standard deviation, number, or mean (range), unless otherwise stated.

PJK, proximal junctional kyphosis.

Table 3. Invasiveness of each procedure

\begin{tabular}{|c|c|c|c|}
\hline Variable & Operation time (min) & Blood loss (mL) & $\%$ Estimated blood volume (\%) \\
\hline \multicolumn{4}{|c|}{ Total rod replacement } \\
\hline$+(n=42)$ & $206.6 \pm 65.3$ & $474.5 \pm 452.2$ & $15.2 \pm 15.0$ \\
\hline$-(n=11)$ & $190.6 \pm 58.4$ & $607.9 \pm 575.8$ & $21.3 \pm 20.2$ \\
\hline$p$-value & 0.463 & 0.415 & - \\
\hline \multicolumn{4}{|c|}{ Bone graft in interbody or osteotomy site } \\
\hline$+(n=21)$ & $247.4 \pm 40.1$ & $729.5 \pm 581.7$ & $23.9 \pm 21.4$ \\
\hline$-(n=32)$ & $174.4 \pm 59.9$ & $353.0 \pm 325.9$ & $11.3 \pm 7.2$ \\
\hline$p$-value & $<0.001^{\text {a) }}$ & $0.004^{\mathrm{al}}$ & - \\
\hline \multicolumn{4}{|c|}{ Elongation to proximal (for PJK) } \\
\hline$+(n=12)$ & $265.8 \pm 48.8$ & $631.2 \pm 494.1$ & $20.4 \pm 19.3$ \\
\hline$-(n=41)$ & $185.1 \pm 55.7$ & $464.4 \pm 472.0$ & $15.2 \pm 15.6$ \\
\hline$p$-value & $<0.001^{\text {a) }}$ & 0.292 & - \\
\hline
\end{tabular}

Values are presented as mean \pm standard deviation.

PJK, proximal junctional kyphosis.

${ }^{\text {a) }}$ Statistically significant. 
Table 4. Re-fracture patients following revision surgery

\begin{tabular}{lcccllll} 
Patient no. & Age (yr) & Sex & Duration between revision to re-rod fracture (mo) & Injury situation & Re-RF site & Bone graft in anterior column \\
\hline 1 & 73 & F & 36 & Traffic accident & Below MR & + \\
2 & 70 & F & 28 & Fall down & Above MR & - \\
\hline 3 & 49 & F & 30 & Fall down & Inside MR & Inside MR & + \\
4 & 52 & M & 47 & Lifting & Inside MR & + \\
\hline 5 & 57 & F & 42 & No injury & & + \\
\hline
\end{tabular}

$\mathrm{RF}$, radiofrequency; $\mathrm{F}$, female; $\mathrm{M}$, male; $\mathrm{MR}$, multi-rod construct.

\begin{tabular}{lrrcc} 
Table 5. Comparison between intraoperative findings and CT findings \\
Bone union on CT & \multicolumn{5}{c}{ Mobility in revision surgery } \\
\cline { 2 - 5 } & + & - & Unknown & Total \\
+ & 8 & 7 & 0 & 15 \\
- & 30 & 4 & 4 & 38 \\
Total & 38 & 11 & 4 & 53
\end{tabular}

$\mathrm{CT}$, computed tomography.

L5/S1 in 10 patients, L3-L5 in eight patients, and above L3 in three patients. A bone graft was placed in the anterior column at the site of the 3-column osteotomy for six patients, posterior lumber interbody fusion was performed for nine patients, and posterolateral fusion was conducted for five patients. The invasiveness of each procedure in revision surgery is shown in Table 3. Surgical invasion for total or partial rod replacement had no significant differences. However, surgical invasion for the procedures with and without an anterior bone graft significantly differed. The replaced rod refractured at an average of 36.3 months after the revision surgery of five patients (Table 4).

Bone union and mobility during the reoperation of 49 patients were compared, but four patients without recorded intraoperative CT findings were excluded. Despite the diagnosis of bone union on CT, instability was observed in eight patients during reoperation. In four patients, CT showed no bone fusion, but the intraoperative findings indicated no mobility. CT examination on bone fusion showed a sensitivity of $78.9 \%$ and a specificity of $63.6 \%$ (Table 5).

\section{Illustrative cases}

\section{1) Patient 2 (Fig. 1)}

A 70-year-old woman was diagnosed with spinal de- formity caused by progressive supranuclear palsy. She underwent a thoracopelvic corrective fusion with an L3 pedicle subtraction osteotomy (Fig. 1A, B). She reported a clicking sound in her back 25 months after the primary surgery, and a rod fracture was observed at the osteotomy site (Fig. 1C, D). Revision surgery was performed with a partial rod replacement and a multirod construct (Fig. 1E, F). A bilateral rod fracture was detected above the T12 pedicle screws following a traumatic fall 28 months after revision surgery (Fig. 1G, H). Re-revision surgery was conducted with a partial rod replacement and multirod construct (Fig. 1I, J).

\section{2) Patient 4 (Fig. 2)}

A 52-year-old man with an iatrogenic deformity underwent a corrective fusion surgery from T10 to the ilium for ilium fusion. A rod fracture was observed at the L3/4 level 8 months after the initial surgery (Fig. 2A, B). Revision surgery was performed using a posterior approach with a total rod replacement, multirod construct, and bone graft in the intervertebral disc (Fig. 2C, D). A rod fracture was found at the L3/4 and L4/5 levels 47 months after revision surgery (Fig. 2E, F). Re-revision surgery was conducted with a total rod replacement and multirod construct (Fig. $2 \mathrm{G}, \mathrm{H})$.

\section{Discussion}

Rod fracture after ASD surgery is a common mechanical complication; however, its treatment has been rarely reported [11]. To the best of our knowledge, our study is the first to show the postoperative clinical results of revision surgery via a posterior-only approach for rod fracture after a corrective fusion for ASD. The incidence of rod fractures following ASD surgery was 21.8\% (88 patients) in this study. A total of 53 patients underwent revision surgery for rod fracture 28.3 months (on average) after 

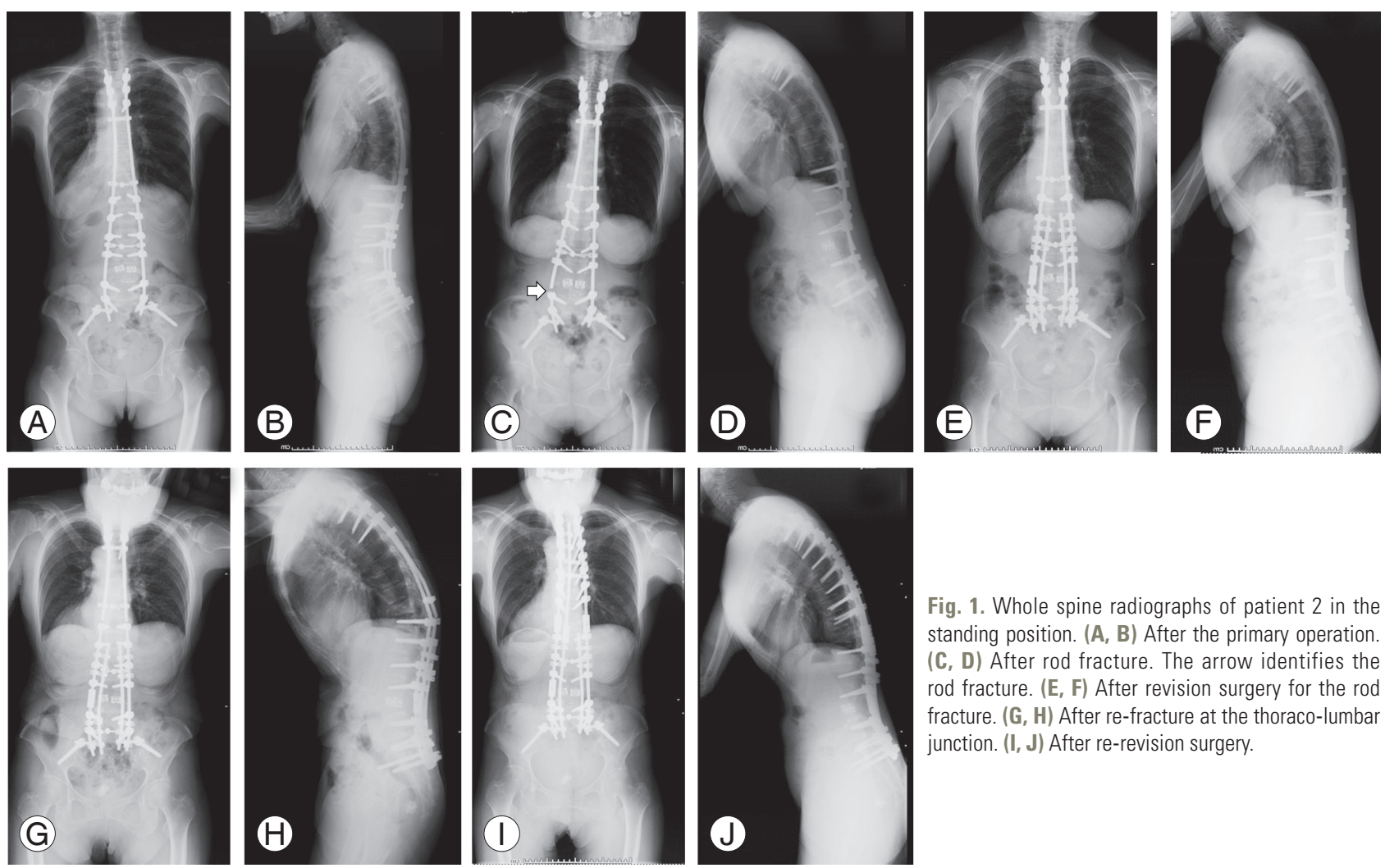

Fig. 1. Whole spine radiographs of patient 2 in the standing position. (A, B) After the primary operation. (C, D) After rod fracture. The arrow identifies the rod fracture. (E, F) After revision surgery for the rod fracture. (G, H) After re-fracture at the thoraco-lumbar junction. (I, J) After re-revision surgery.
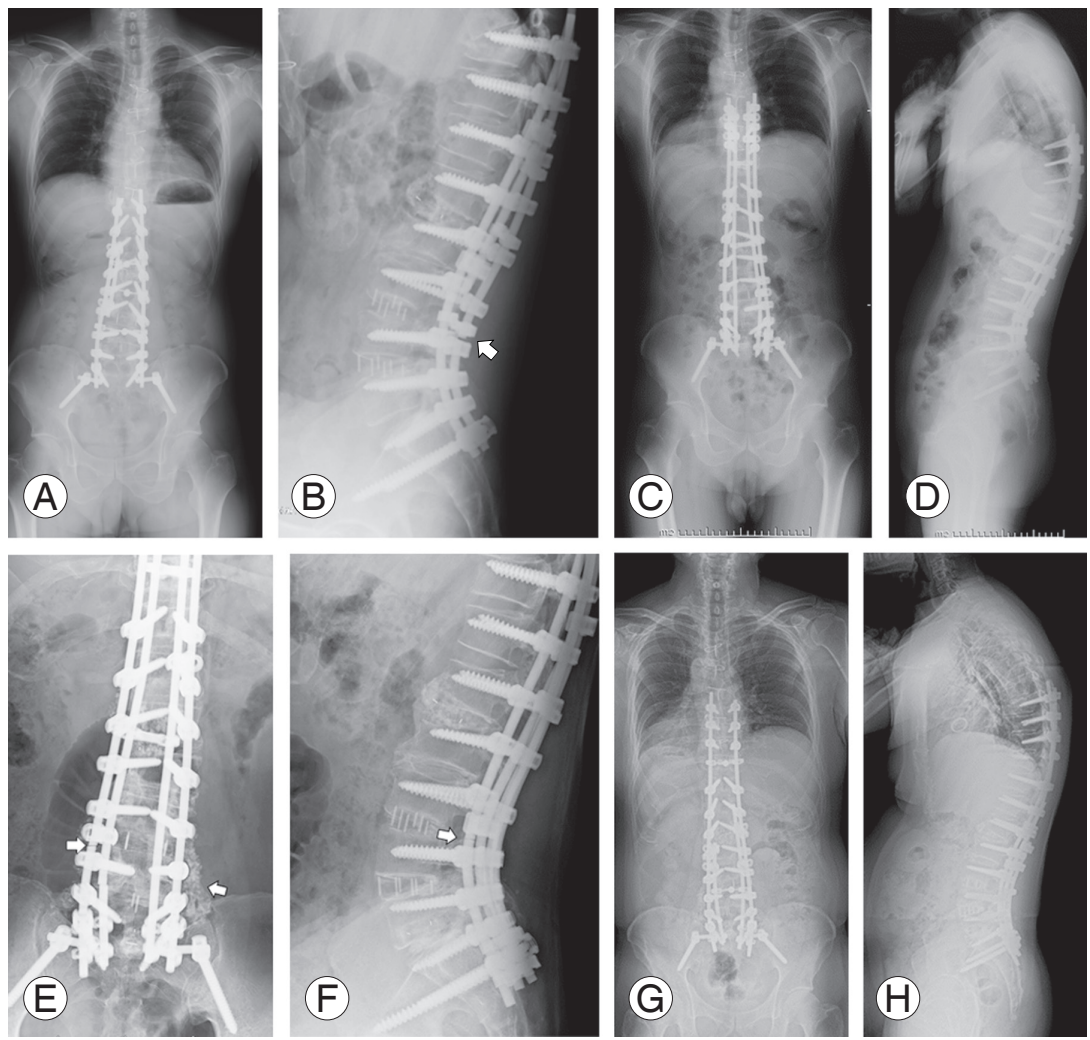

Fig. 2. Whole spine radiographs of patient 4 in the standing position. (A, B) After rod fracture. The arrow identifies the rod fracture. (C, D) Revision surgery for the rod fracture. (E, F) Re-fracture at the $L 3 / 4$ and L4/5 levels. The arrows identify the rod fracture. (G, H) After re-revision surgery. 
the primary surgery. After the revision surgery, the symptoms of all the patients were alleviated. During an average of 46.7-month follow-up, only five patients (9.4\%) refractured their replaced rods. Revision surgery showed good clinical results regarding symptoms and refracture. Luca et al. [10] reported eight patients who underwent revision surgery for rod fracture via a combined posteroanterior approach or a lateral transpsoas combined approach. They reported a mean operative time (including all stages of the procedure) of 7.2 hours, and one of the eight patients experienced a second rod fracture only 8 months after reoperation. These findings suggested that the surgical method using the posterior-only approach could sufficiently treat rod fractures.

Whether anterior reconstruction is necessary for all cases is controversial. Daniels et al. [14] showed that rod fracture occurs in $9.5 \%$ of patients with apparently solid radiographic fusion after ASD surgery, and 21.1\% of these patients required revision surgery. Instruments may fail even in cases with evidence of apparent fusion on plain radiographs. Our study also found some cases with intraoperative findings that differ from CT findings; consequently, diagnosing nonunion from CT examination was difficult. Luca et al. [10] performed anterior reconstruction in all cases. In our cases, a bone graft was placed in the anterior column of 21 of the 53 patients via the posterior approach; however, four of the 21 patients with a bone graft in the anterior column refractured their rods. Of the 25 patients without an anterior bone graft, only one refractured a rod. In addition, refracture occurred at a different site from the initial rod fracture in two of the five cases. These results showed that refracture occurs even after anterior column reconstruction. Anterior and transforaminal interbody fusion procedures at the caudal levels of long spinal deformity constructs are associated with low bilateral rod fracture rates and do not differ in the rate of revision surgery between the two groups [15]. Further, the prevalence of rod fractures is not significantly different between anterior and transforaminal groups [15]. Although we performed anterior reconstruction for patients with anterior bone defects using the posterior approach, no failure occurred in most instances. Anterior reconstruction or bone grafting may be unnecessary for patients without massive bone defects in the anterior column.

Multirods are installed to prevent rod fracture after ASD surgery [8,16-22]. Through a multirod technique, stress on the rod can be avoided [23,24], and the degree of rod breakage can be reduced. However, rod fractures were detected in $11.8 \%$ of patients with a multirod construct after ASD surgery [21]. They mostly occur at the lumbosacral junction or an adjacent level [11]. When a multirod is used, stress concentrates on its uncovered part. As such, a rebreakage is highly likely to occur at the edge of a multirod [22]. Therefore, our recommendation is that multirods should be installed such that they cover the lumbosacral joint (the connector of the iliac screw).

Full or partial replacement of all rods is controversial. One method involves reinforcing a rod by using a connector to bridge its fractured part, but this method is complicated, and transforming the fractured rod into a multirod construct is difficult. Connecting the rods with a vertical connector is a difficult task during surgery, and removing the screw at the bent portion of the lumbosacral junction or osteotomy site may be necessary. Although a connection can be established with a horizontal connector, this procedure causes problems such as difficulty in performing a multirod procedure and possible use of too many rods. Therefore, we often remove all rods at once and replace them. In this study, no significant changes in surgical invasion were observed even if all rods were replaced.

This study has several limitations: retrospective design, single-center setting, small number of patients, and lack of clinical evaluation such as patient-reported outcome measurements before and after revision surgery. A longer follow-up period is required to determine the long-term results of reconstruction using multirods. The most important limitation is that the present surgical results are not compared with other reconstruction method results. Although good results of the reoperation were obtained in this study, efforts should be devoted to reducing the occurrence of rod fractures.

\section{Conclusions}

Reoperations for rod fractures after ASD were successfully performed clinically. Surgical procedures using a posterior-only approach with a multirod that covers the lumbosacral joint can prevent the refracture of replacement rods in short to medium term. Further details are needed to validate the results of these methods with multirod constructs in studies that examine medium- to longterm outcomes. 


\section{Conflict of Interest}

Yu Yamato and Shin Oe work at a donation-endowed laboratory in the Division of Geriatric Musculoskeletal Health. The other authors have no potential conflict of interest relevant to this article.

\section{Acknowledgments}

The authors would like to express great appreciation to Ms. Nao Kuwahara, Ms. Tomoe Mabuchi, Mr. Taku Nagao, and Mr. Tomokazu Suzuki, who are secretaries in the Department of Orthopedic Surgery, for their contribution to data correction.

\section{Funding}

Yu Yamato and Shin Oe received financial support from Medtronic Sofamor Danek Inc., Japan, Medical Dynamic Marketing Inc., and Meitoku Medical Institution Jyuzen Memorial Hospital. The other authors did not receive support from any organization for the submitted work.

\section{Author Contributions}

Conception or design of the work: Yu Yamato, Yukihiro Matsuyama; acquisition, analysis, or interpretation of data for the work: Yu Yamato, Tomohiko Hasegawa, Go Yoshida, Tomohiro Banno, Hideyuki Arima, Shin Oe, Yuki Mihara, Hiroki Ushirozako, Tomohiro Yamada, Yuh Watanabe, Koichiro Ide, Keiichiro Nakai, Kenta Kurosu; drafting of the work: Yu Yamato; critical revision for important intellectual content: Tomohiko Hasegawa, Go Yoshida, Tomohiro Banno, Shin Oe, Hideyuki Arima, Yuki Mihara, Hiroki Ushirozako, Tomohiro Yamada, Yuh Watanabe, Koichiro Ide, Keiichi Nakai, Kenta Kurosu, Yukihiro Matsuyama; and final approval of the version to be published: all authors.

\section{References}

1. Soroceanu A, Diebo BG, Burton D, et al. Radiographical and implant-related complications in adult spinal deformity surgery: incidence, patient risk factors, and impact on health-related quality of life. Spine (Phila Pa 1976) 2015;40:1414-21.

2. DeWald CJ, Stanley T. Instrumentation-related com- plications of multilevel fusions for adult spinal deformity patients over age 65: surgical considerations and treatment options in patients with poor bone quality. Spine (Phila Pa 1976) 2006;31(19 Suppl):S144-51.

3. Kim HJ, Yang JH, Chang DG, et al. Proximal junctional kyphosis in adult spinal deformity: definition, classification, risk factors, and prevention strategies. Asian Spine J 2021 Apr 30 [Epub]. https://doi. org/10.31616/asj.2020.0574.

4. Smith JS, Shaffrey CI, Ames CP, et al. Assessment of symptomatic rod fracture after posterior instrumented fusion for adult spinal deformity. Neurosurgery 2012;71:862-7.

5. Akazawa T, Kotani T, Sakuma T, Nemoto T, Minami S. Rod fracture after long construct fusion for spinal deformity: clinical and radiographic risk factors. J Orthop Sci 2013;18:926-31.

6. Smith JS, Shaffrey E, Klineberg E, et al. Prospective multicenter assessment of risk factors for rod fracture following surgery for adult spinal deformity. J Neurosurg Spine 2014;21:994-1003.

7. Barton C, Noshchenko A, Patel V, Cain C, Kleck C, Burger E. Risk factors for rod fracture after posterior correction of adult spinal deformity with osteotomy: a retrospective case-series. Scoliosis 2015;10:30.

8. Hamilton DK, Buza JA 3rd, Passias P, et al. The fate of patients with adult spinal deformity incurring rod fracture after thoracolumbar fusion. World Neurosurg 2017;106:905-11.

9. Lertudomphonwanit T, Bridwell KH, Kelly MP, et al. Relationship of the character of rod fractures on outcomes following long thoracolumbar fusion to the sacrum for adult spinal deformity. Spine J 2020;20:1452-63.

10. Luca A, Lovi A, Galbusera F, Brayda-Bruno M. Revision surgery after PSO failure with rod breakage: a comparison of different techniques. Eur Spine J 2014;23 Suppl 6:610-5.

11. Yamato Y, Hasegawa T, Kobayashi S, et al. Treatment strategy for rod fractures following corrective fusion surgery in adult spinal deformity depends on symptoms and local alignment change. J Neurosurg Spine 2018;29:59-67.

12. Kim YJ, Bridwell KH, Lenke LG, Rhim S, Cheh G. Pseudarthrosis in long adult spinal deformity instrumentation and fusion to the sacrum: prevalence and risk factor analysis of 144 cases. Spine (Phila Pa 1976) 
2006;31:2329-36.

13. Kanda Y. Investigation of the freely available easy-touse software 'EZR' for medical statistics. Bone Marrow Transplant 2013;48:452-8.

14. Daniels AH, DePasse JM, Durand W, et al. Rod fracture after apparently solid radiographic fusion in adult spinal deformity patients. World Neurosurg 2018;117:e530-7.

15. Adogwa O, Buchowski JM, Lenke LG, et al. Comparison of rod fracture rates in long spinal deformity constructs after transforaminal versus anterior lumbar interbody fusions: a single-institution analysis. J Neurosurg Spine 2019:1-8.

16. Shen FH, Harper M, Foster WC, Marks I, Arlet V. A novel "four-rod technique" for lumbo-pelvic reconstruction: theory and technical considerations. Spine (Phila Pa 1976) 2006;31:1395-401.

17. Kelly BP, Shen FH, Schwab JS, Arlet V, Diangelo DJ. Biomechanical testing of a novel four-rod technique for lumbo-pelvic reconstruction. Spine (Phila $\mathrm{Pa}$ 1976) 2008;33:E400-6.

18. Hyun SJ, Lenke LG, Kim YC, Koester LA, Blanke KM. Comparison of standard 2-rod constructs to multiple-rod constructs for fixation across 3-column spinal osteotomies. Spine (Phila Pa 1976) 2014;39:1899-904.
19. Merrill RK, Kim JS, Leven DM, Kim JH, Cho SK. Multi-rod constructs can prevent rod breakage and pseudarthrosis at the lumbosacral junction in adult spinal deformity. Global Spine J 2017;7:514-20.

20. Jung JM, Hyun SJ, Kim KJ, Jahng TA. Rod fracture after multiple-rod constructs for adult spinal deformity. J Neurosurg Spine 2019:1-8.

21. Guevara-Villazon F, Boissiere L, Hayashi K, et al. Multiple-rod constructs in adult spinal deformity surgery for pelvic-fixated long instrumentations: an integral matched cohort analysis. Eur Spine J 2020;29:886-95.

22. Yamato Y, Hasegawa T, Togawa D, et al. Long additional rod constructs can reduce the incidence of rod fractures following 3-column osteotomy with pelvic fixation in short term. Spine Deform 2020;8:481-90.

23. Scheer JK, Tang JA, Deviren V, et al. Biomechanical analysis of revision strategies for rod fracture in pedicle subtraction osteotomy. Neurosurgery 2011;69:164-72.

24. Luca A, Ottardi C, Sasso M, et al. Instrumentation failure following pedicle subtraction osteotomy: the role of rod material, diameter, and multi-rod constructs. Eur Spine J 2017;26:764-70. 